\title{
A new cosmic ray observation at Syowa Station in the antarctic
}

\author{
Chihiro Kato ', Wataru Kihara', Yukino Ko', Kazuoki Munakata ', Shin-ichi Uchida', Sou Kaimi', \\ Ryuho Kataoka ${ }^{\circledR 2}$, Akira Kadokura- ${ }^{2}$, Paul Evenson ${ }^{3}{ }^{3}$, Yoshiaki Nakamura ${ }^{4}$, Kiyoka Murase ${ }^{\circledR 5}$, \\ Shoko Miyake ${ }^{\circledR}$ \\ Correspondence \\ 1Physics Department, Shinshu University, Matsumoto, Japan, ckato@shinshu-u.ac.jp \\ ${ }^{2}$ National Institute of Polar Research, Tokyo, Japan \\ ${ }^{3}$ Bartol Research Institute, Department of Physics and Astronomy, University of Delaware, USA \\ ${ }^{4}$ Key Laboratory of Particle Astrophysics, Institute of High Energy Physics, Chinese Academy of Sciences, \\ Beijing, China \\ ${ }^{5}$ Department of Polar Science, The Graduate University for Advanced Studies, SOKENDAl, Hayama, Japan \\ ${ }^{6}$ Department of Electrical and Electronic Systems Engineering, National Institute of Technology, \\ Ibaraki College, Hitachinaka, Japan
}

\section{OPEN ACCESS}

This work is published under the Creative Commons Attribution 4.0 International licence (CC BY 4.0). Please note that individual, appropriately marked parts of the work may be excluded from the licence mentioned or may be subject to other copyright conditions.

If such thirdpanty mans. If under the Creative Commons license, any copying, editing or public reproduction is only permitted with the prior consent of the respective copy right owner or on the basis of relevan legal authorization regulations.

\section{Introduction}

It is well known that cosmic ray (CR) network observation is the only way to derive data for CR anisotropy analysis of space weather phenomena. Two network observations on the globe are in operation. One is Space Ship Earth (SSE) Neutron Monitor (NM) network, which observes atmospheric neutrons with energy of about $10 \mathrm{GeV}$ as median energy. The other is Global Muon Detector Network (GMDN), Muon Detector (MD) network, which observes secondary muons with the energy of about $60 \mathrm{GeV}$ as median energy. These two observation projects have attained some progress in space weather study individually (e.g. Bieber et.al. 2004; Munakata et.al. 2018). Since the SSE and the GMDN observe different energy regions, integration analysis of these two network observation data would provide valuable information for space weather study. For this purpose, it is necessary to observe the same direction at the same time with MD and NM for normalization between these two network observation projects. 


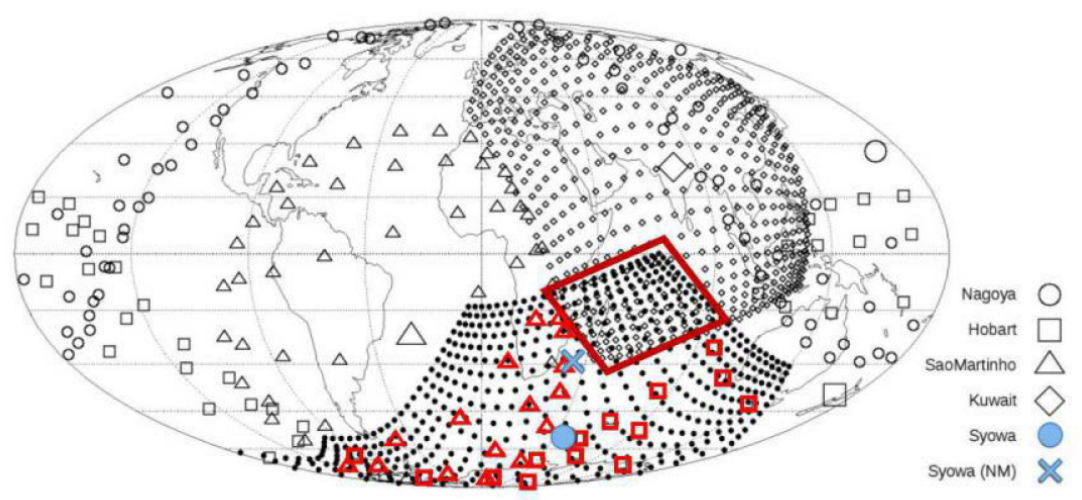

Figure 1: FOV of the GMDN and Syowa MD, and asymptotic direction of the Syowa NM. Symbols indicate asymptotic viewing directions of incident CRs with median rigidity for every directional component of the GMDN, Syowa MD, and Syowa NM. Syowa MD and Syowa NM observe the same direction. Superposed red mark indicates overlapping direction between the GMDN and Syowa MD.

The Syowa Station is located on East Ongul Island, Lützow-Holm Bay and the GMDN stations are located at Nagoya (Japan), Hobart (Australia), Sao Martinho (Brazil), and Kuwait City (Kuwait). The distribution of the asymptotic viewing directions of incident CRs with median rigidity observed by each directional channel at the GMDN station and Syowa MD, and Syowa NM is shown in figure 1. Open symbols are for the GMDN stations and the closed symbol is for the Syowa MD. Large symbols indicate vertical directional channels at each station. The blue cross symbol indicates the asymptotic viewing direction of Syowa NM. The asymptotic direction of Syowa NM is in the FOV of Syowa MD. Thus, Syowa NM and Syowa MD can observe the same direction. Furthermore, there are overlapping viewing directions between Syowa MD and GMDN, which are shown by superposed red symbols. Therefore, Syowa CR observation system can be used not only for normalization between the GMDN and the SSE but also among the GMDN stations. The observation was started in February 2018. In this paper, the new CR observation system at Syowa Station is described.

\section{New detector system}

The detector system was installed by the 59th Japanese Antarctic Research Expedition (JARE59) from the end of 2017 to the beginning of 2018. The system is located at (latitude, longitude, altitude) $=$ $\left(-69.01^{\circ}, 39.59^{\circ}, 24.7 \mathrm{~m}\right)$. Characteristics of the detectors are summarized in table 1.

\begin{tabular}{|c|c|c|c|c|c|}
\hline $\begin{array}{l}\text { Directional } \\
\text { Channel }\end{array}$ & $\begin{array}{l}\text { Cutoff Rigidity Pc } \\
\text { [GV] }\end{array}$ & $\begin{array}{l}\text { Median Rigidity } \\
\text { Pm [GV] }\end{array}$ & $\begin{array}{l}\text { Asymptotic } \\
\text { directions@Pm }\end{array}$ & $\begin{array}{l}\text { Correction } \\
\text { coefficient } \beta \\
{[\% / \mathrm{hPa}]}\end{array}$ & $\begin{array}{l}\text { Average Count } \\
\text { rate } \times 10^{4} \\
\text { [Counts } / \mathrm{hr} \text { ] }\end{array}$ \\
\hline \multicolumn{6}{|l|}{ Syowa NM } \\
\hline Omni & 0.4 & 15.2 & $30 \mathrm{~S} 38 \mathrm{E}$ & -0.76 & 31.8 \\
\hline \multicolumn{6}{|l|}{ SyowaMD } \\
\hline V & 2.5 & 53.6 & $56 \mathrm{~S} 53 \mathrm{E}$ & -0.16 & 26.9 \\
\hline$N$ & 2.8 & 58.5 & $29 \mathrm{~S} 35 \mathrm{E}$ & -0.16 & 3.8 \\
\hline S & 2.8 & 58.5 & 70S115E & -0.16 & 3.8 \\
\hline
\end{tabular}




\begin{tabular}{|l|l|l|l|l|l|}
\hline$E$ & 2.8 & 58.5 & $32 S 81 E$ & -0.16 & 6.4 \\
\hline W & 2.8 & 58.5 & $60 S 8 \mathrm{~W}$ & -0.16 & 6.4 \\
\hline NE & 3.2 & 63.6 & $16 S 60 \mathrm{E}$ & -0.15 & 1.0 \\
\hline NW & 3.2 & 63.6 & $34 S 4 E$ & -0.16 & 1.0 \\
\hline SE & 3.2 & 63.6 & $42 S 110 \mathrm{E}$ & -0.16 & 1.0 \\
\hline SW & 3.2 & 63.6 & $80 S 77 \mathrm{~W}$ & -0.16 & 1.0 \\
\hline
\end{tabular}

Table 1: Characteristics of Syowa NM and Syowa MD.

The detectors are assembled in two refrigeration containers as shown in figure 2. Schematic views of the frame of the detector system are also shown in figure 2. Six NM64 and sixty proportional counter tubes (PCTs), which diameter is $10 \mathrm{~cm}$, are used for NM and MD, respectively. Three NM64 are housed in a polyethylene box after covering individually with a thin polyethylene shell and lead at the top of the frame.
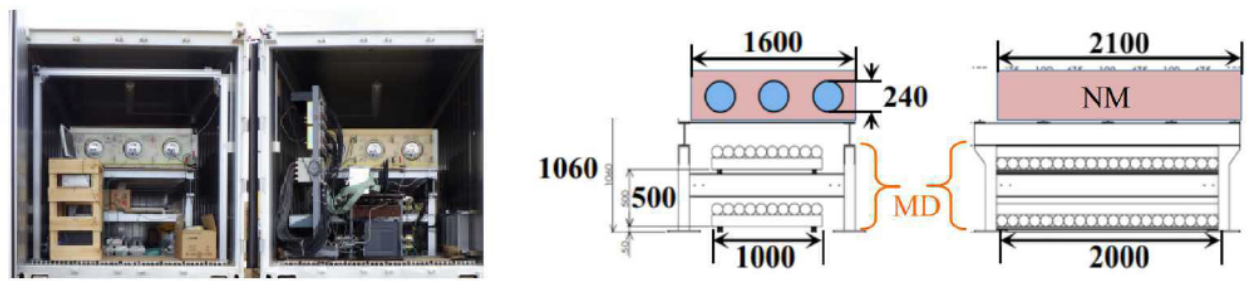

Figure 2: Left: Inside of the refrigeration containers.

Right: Schematic view of the detector system. Numbers are in $\mathrm{mm}$.

The MD is placed under the NM. There are PCTs of two different lengths, which are $1 \mathrm{~m}$ and 2 $\mathrm{m}$, to fit in the container. Since MD records directional counts, PCTs are set into four layers. How to determine the incident direction by 4 fold coincidence can be seen in figure 3 . Layers of $1 \mathrm{~m}$ and $2 \mathrm{~m}$ PCTs are paired up in an orthogonal arrangement. The upper and lower pair of the PCTs give a set of $(x, y)$ coordinates for the incident muon. Thus incident direction can be determined. The $\mathrm{x}$ - and $\mathrm{y}$-directions are following the long and short sides, respectively. The $\mathrm{y}$-direction is off from North by 20.06 degrees at a clockwise angle. It should be noted that MD is assembled in only one container because of the budget constraints. The observation started on 1 February 2018.

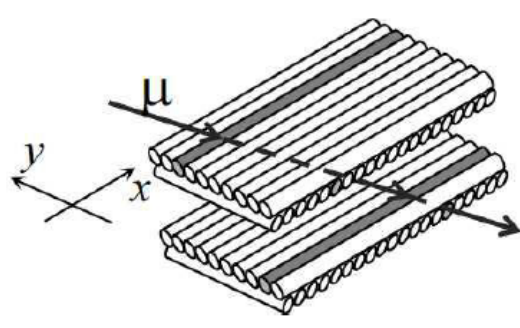

Figure 3: Conceptual diagram of 4 fold coincidence of Syowa MD. X-, $y$-directions are following the long and short sides, respectively. 
Because the containers are outside, the inside temperature of the container dropped close to -20 degrees Celsius during the first winter. One of the data taking PC failed once during this winter by an unknown cause. But to protect the PC and the other electronics, a heating system was installed in each container to maintain the interior temperature of the containers after one year of operation. The PCs are also covered by an insulator. This system maintains the interior temperature of the containers about $0-7$ degrees and around the PCs about $10-17$ degrees.

\section{Data flow of the detector system}

Figure 4 is showing the data flow of these observations. The NM records integrated counts every minute. The MD records counts/minute and counts/10minutes data. Recorded data is automatically transferred to the data server at Shinshu University, Japan through satellite communication and the internet. Frequencies of the data transfer are once a day for NM and every hour for MD. Hourly counts data are created automatically from the counts/10minutes data on the data server after the correction of the atmospheric pressure effect. It is planned to provide NM data to World Data Center (WDC) and Neutron Monitor Data Base (NMDB). Hourly count data of NM and MD's vertical channel are provided to the QL server at the National Institute of Polar Research in Japan'.

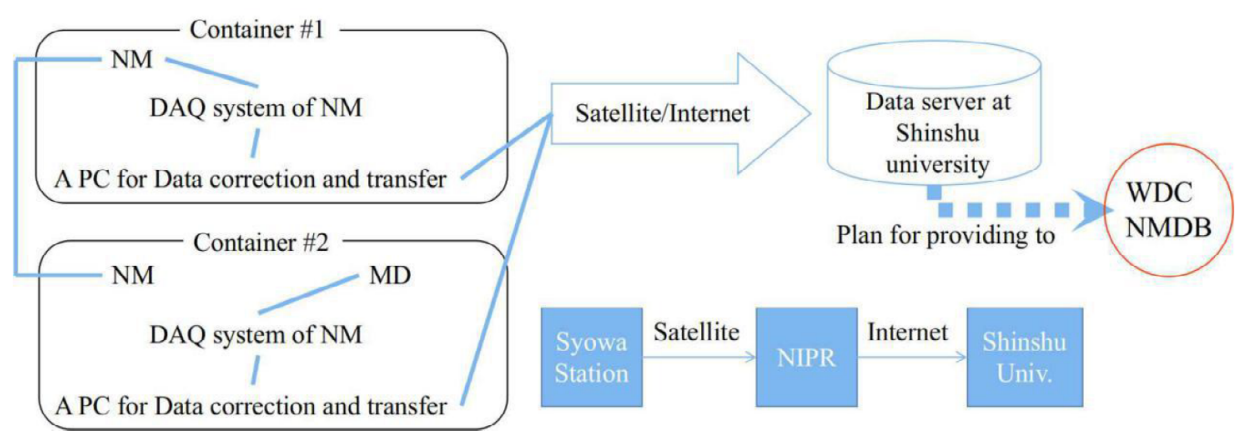

Figure 4: Data-flow diagram of SyowaMD and SyowaNM. Data is transferred via satellite communication and the internet and stored in the data server at Shinshu University.

\section{Summary}

Since observation started on 1 February 2018, observation is stable and operation rates are $99 \%$ for MD and $91 \%$ for NM. The lower rate for NM is because of PC trouble in the first winter and in the middle of 2020. The later trouble was related to the internet connection. The daily averaged count of NM and of the vertical channel of MD are shown in figure 6 from 2018.2 to 2020.7. Deviation from average is plotted. Data accumulating for two and half years shows temperature effect on the muon count as expected. The neutron count rate is consistent with South Pole NM. This is one of the collateral evidence that Syowa MD and Syowa NM are functioning correctly.

We conclude that the new CR observation system is working correctly and stable to carry out long-term observation. The new system will provide data for integrated analysis of SSE and GMDN and for a detailed study of the difference of atmospheric effect on NM and MD. 


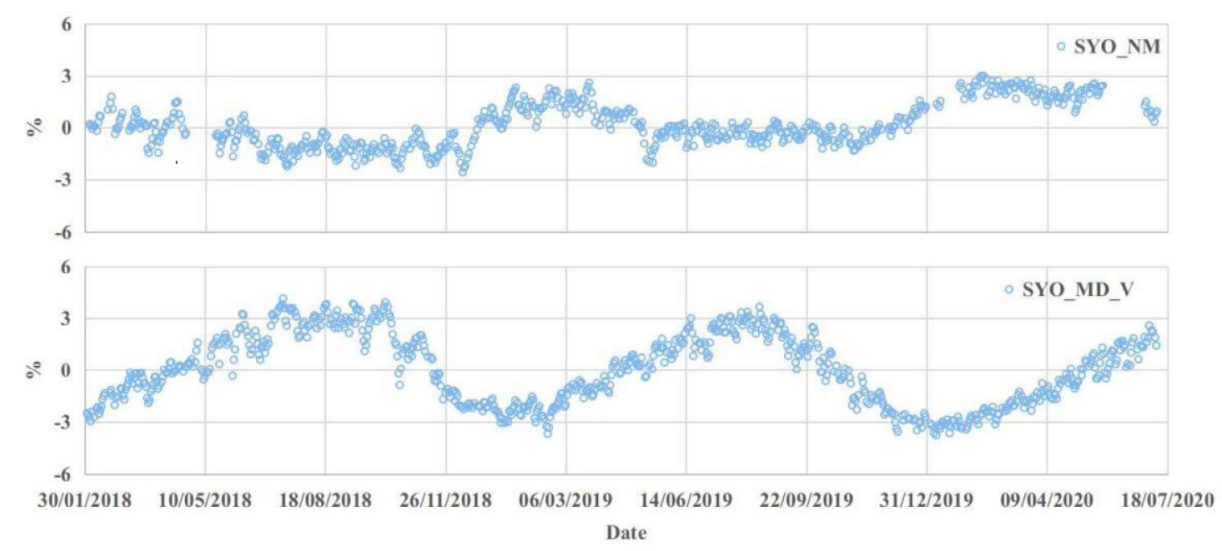

Figure 5: Daily averaged count data of SyowaNM (upper panel) and SyowaMD: vertical channel(lower panel). The operation rates are $91 \%$ for NM and $99 \%$ for MD. The atmospheric temperature effect can be seen on MD data.

\section{References}

Bieber, J., Evenson, P., Dröge, W., and Pyle, R., 2004, Spaceship Earth Observations of the Easter 2001 Solar Particle Event, Astrophys. J., DOI: https://doi.org/10.1086/381801

Munakata, K., Kozai, M., Evenson, P., Kuwabara, T., et. al., 2018, Cosmic-Ray Short Burst Observed with the Global Muon Detector Network (GMDN) on 2015 June 22, Astrophys. J., DOI: https://doi.org/10.3847/1538-4357/aacdfe

\section{Questions and answers}

Question: Does the lead above the MD affect the incident direction by scattering?

Answer: It is important to estimate with MC simulation because it is unclear for Syowa MD. Currently, several papers are found in which scattering of muon in thin lead target is reported by accelerator beam experiment (e.g. Paoloni, A., Pupilli, F., 2015, IEEE Transactions on Nuclear Science, DOI: https://doi.org/10.1109/ TNS.2015.2473674).

Alayman: How can the directions be the same, given that the median energies differ between a NM and a MD located at the same place?

Answer: Geomagnetic field at high latitude does not make a significant difference on orbit deflections of CR for NM and MD unlike in the case of lower latitude. 